\title{
Fontan-associated liver disease: Is it all about hemodynamics?
}

\author{
David Kalfa, MD, PhD
}

From the Division of Cardiac, Thoracic, and Vascular Surgery, Section of Pediatric and Congenital Cardiac Surgery, New York Presbyterian-Morgan Stanley Children's Hospital, Columbia University Medical Center, New York, NY.

Disclosures: Author has nothing to disclose with regard to commercial support.

Received for publication March 21, 2018; accepted for publication March 27, 2018; available ahead of print May 3, 2018.

Address for reprints: David Kalfa, MD, PhD, New York Presbyterian Hospital CHONY, CUMC, 3959 Broadway, New York, NY 10032 (E-mail: dk2757@ cumc.columbia.edu).

J Thorac Cardiovasc Surg 2018;156:276-7

$0022-5223 / \$ 36.00$

Copyright (C) 2018 by The American Association for Thoracic Surgery

https://doi.org/10.1016/j.jtcvs.2018.03.104

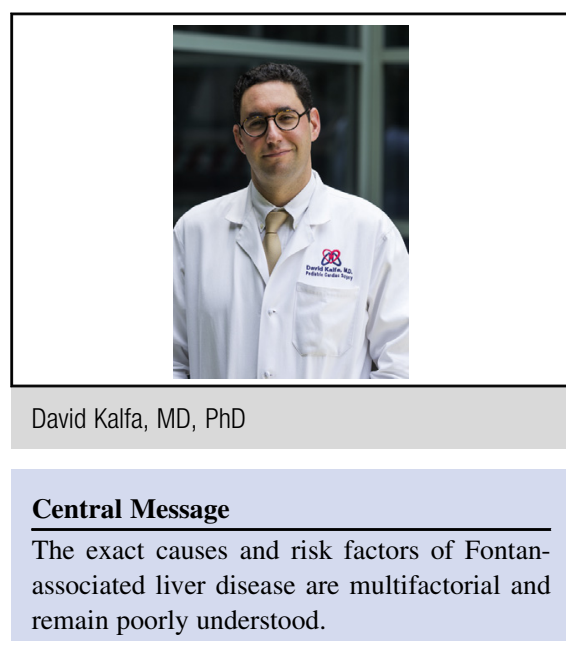

The study by Trusty et al, ${ }^{1}$ in this issue of The Journal of Thoracic and Cardiovascular Surgery, aims at investigating detailed cardiac magnetic resonance (CMR)-derived flow dynamics and total cavopulmonary connection (TCPC) energetics as potential risk factors for hepatic fibrosis in Fontan patients. Fontan-associated liver disease (FALD) has been a well documented complication for decades ${ }^{2}$ and is now recognized as a progressive process affecting all Fontan survivors. The exact causes and risk factors of FALD remain poorly understood.

Trusty et al ${ }^{1}$ tackled this controversial topic by combining CMR, computational fluid dynamics, cardiac catheterization, and liver biopsy data to investigate potential relationships between hepatic fibrosis and Fontan hemodynamics. They showed that liver fibrosis was found to be related to global metrics (inferior vena cava [IVC] flow, cardiac output) rather than to local TCPC hemodynamics and efficiency. One of the strengths of this study is that the authors had the opportunity to analyze contemporaneous liver biopsy data in conjunction with detailed blood flow characteristics in each patient.

Interestingly and surprisingly, the study findings do not support the notion that finer aspects of flow hemodynamics through the TCPC mitigate the presence of liver fibrosis. The authors found that Fontan energetics were not associated with hepatic fibrosis, and state that Fontan pathway architecture and TCPC inefficiency is not the key factor contributing to the degree of fibrosis. Nevertheless, it is important to note that this study did not include patients with clinically significant TCPC pathway obstruction. Thus, the pediatric cardiac medical and surgical community should keep in mind that, despite the findings of this valuable CMR/computational fluid dynamics-based study, an optimal Fontan without obstruction remains the very basic goal that we all need to achieve to optimize long-term outcomes for these patients, and that an aggressive diagnostic and therapeutic approach for Fontan obstruction remains warranted during the whole life of these patients.

See Article page 267

Stating that the Fontan energetics are not associated with hepatic fibrosis, the authors hypothesize that the positive association seen between the degree of hepatic fibrosis and the IVC flow rate and cardiac output could be related to hepatic arterialization (and subsequent increased hepatic venous return). However, despite the quality of this study from Yoganathan's engineering group and Philadelphia clinical group, we still do not understand if the increased IVC flow is the cause or consequence of the liver fibrosis. Moreover, all computational simulations performed in this study used rigid walls, which obviously is a significant limitation to truly reflect the fluid hemodynamics in these complex patients. Thus, in the absence of data in favor of this "hepatic hyperarterialization" hypothesis (hypervascular nodularity was not assessed on CMR in this study), the underlying mechanisms for the interindividual variability and a higher susceptibility for developing Fontan-related liver fibrosis remain unknown. In the current era of personalized and precision medicine, genetic roots to this FALD susceptibility should be investigated through multi-institutional initiatives such as the Pediatric Cardiac Genomics Consortium. ${ }^{3}$ We could envision that genetic variants present in the congenital heart disease patient population might be identified and used to predict the development of FALD in the Fontan population.

\section{References}

1. Trusty P, Wei Z, Rychik J, Russo PA, Surrey LF, Goldberg DJ, et al. Impact of hemodynamics and fluid energetics on liver fibrosis after Fontan operation. J Thorac Cardiovasc Surg. 2018;156:267-75. 
2. Lemmer JH, Coran AG, Behrendt DM, Heidelberger KP, Stern AM. Liver fibrosis (cardiac cirrhosis) five years after modified Fontan operation for tricuspid atresia. J Thorac Cardiovasc Surg. 1983;86:757-60.
3. Hoang TT, Goldmuntz E, Roberts AE, Chung WK, Kline JK, Deanfield JE, et al. The Congenital Heart Disease Genetic Network Study: cohort description. PLoS One. 2018;13:e0191319. 